\title{
Self-pop-up Cylindrical Structure by Global Heating
}

\author{
Shuhei Miyashita, Cagdas D. Onal, and Daniela Rus
}

\begin{abstract}
In this study, we demonstrate a new approach to autonomous folding for the body of a 3D robot from a 2D sheet using heat. We approach this challenge by folding a $0.27 \mathrm{~mm}$ sheet-like material into a structure. We utilize the thermal deformation of a contractive sheet sandwiched by rigid structural layers. During this "baking" process, the heat applied on the entire sheet induces contraction of the contracting layer and, thus, forms an instructed bend in the sheet. To attain the targeted folding angles, the V-fold Spans method is used. The targeted angle $\theta_{\text {out }}$ can be kinematically encoded into crease geometry. The realization of this angle in the folded structure can be approximately controlled by a contraction angle $\theta_{i n}$. The process is non-reversible, is reliable, and it is relatively fast. Our method can be applied simultaneously to all the folds in multi-creased origami structures. We demonstrate the use of this method to create a light-weight mobile robot.
\end{abstract}

\section{INTRODUCTION}

The remarkable capabilities of bio-entities in developing a morphology is called morphogenesis. As robots enter our daily life and become more ubiquitous, demands on the instant-fabrication of robots that can morph and are adaptive to respective environments increase. This is a challenge that has been tackled in modular robotics, where a robot's body consisting of multiple modules reconfigures and adapts to environmental changes [1]-[5], or in a similar stream but attempting to handle highly stochastic situations in a bottomup manner [6]-[9], or a folding manner [10]. A unique avenue focusing on extending a robot's physical capabilities is taken by [11], where the robot can print and use a threedimensional tool.

Recently, the folding-based creation of robot bodies from sheet-like materials has shown noticeable accomplishments [12]-[14]. The methods employed in these works make frequent use of origami-inspired foldings to create light-weight mechanics. Complex bodies can be folded from a laser-cut single sheet of paper [15].

This paper makes the following contributions:

1) a model, design, and method for designing flat origami structures that can self-fold in a controlled way using global heating

2) an instantiation of this approach to create a self-folding cylindrical-shaped robot and extensive experimental characterization

S. Miyashita and D. Rus are with the Computer Science and Artificial Intelligence Laboratory, MIT,32 Vassar street, Cambridge, MA, 02139, USA \{shuheim|rus\}@csail.mit.edu

C. D. Onal is with the Mechanical Engineering Department, Worcester Polytechnic Institute, Worcester, MA 01609, USA cdonal@wpi.edu

This research was conducted in the Distributed Robotics Laboratory at CSAIL, MIT. Support for this work has been provided partially by NSF grants 1240383 and 1138967, and the Swiss National Science Foundation Fellowship Grant PBZHP2-133472.

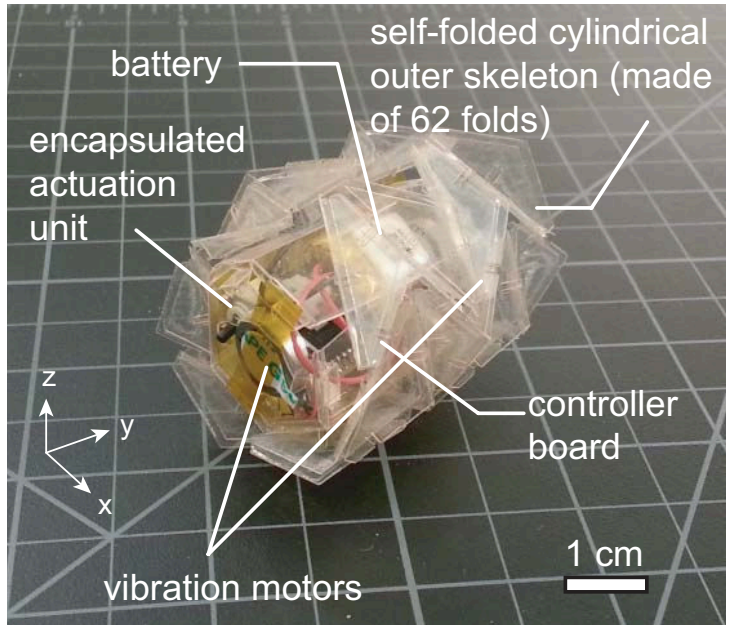

Fig. 1: Outlook of self-folded outer skeleton with a modularized actuation unit.

The key insight is to use a flat sheet sandwiched by rigid structural layers. The structure of the hard layers determines the target angles that are achievable on each structural edge, which determine, in turn, the final geometry of the selffolded object. Next, we impose an origami crease pattern that was designed by hand and added to the robot's body using a laser cutter. Finally, we suspend the sheet in an oven. The self-folding process takes approximately 5 minutes at $65^{\circ} \mathrm{C}$. The resulting shape is a textured cylinder. We add to this cylinder a printed circuit, supporting electronics, and vibration motors, and configure a locomotion robot. The robot can perform locomotion on a plane by altering the speeds of vibration motors (Fig. 1). Creating robot bodies by self-folding is fast, easy, and inexpensive.

\section{Self-FOlding Method}

One of the requisite competencies for the robots that morph from a sheet structure is the self-folding capability. Various attempts for automating folding processes have been attempted [16], using Shape Memory Alloys [17], a contraction sheet combined with Joule heating [18], light illumination for heat transfer [19], [20], pneumatic actuation coupled with an elastic body [21], or hydrogel expansion [22]. Nevertheless, few approaches have attempted to achieve the selffolding of large creased forms with a high degree of accuracy, and demonstrated a realistic fabrication time compared with the folding time.

The self-folding methodology described in this paper consists of the following steps. 
1) We model and derive a method for causing a bend on one edge of a flat body structure created by layering a contraction sheet and structural sheets and using global heat (SectionII-A).

2) We develop a method for designing $2 \mathrm{D}$ planar bridges and gaps in a layered sheet that enables multiple edges to be folded in parallel using heat to create different geometries.

3) We compute and control the angles to be folded using steps 1) and 2); the material and geometry of the creases determine the size of the angle that can be folded (Section II-B).

4) We design a specific crease pattern to test the selffolding approach we advocate; this pattern can be folded reliably as a complete cylinder with patterned faces (Section III-A).

5) We integrate electronics and actuators in the self-folded cylindrical body to create a light-weight mobile robot and demonstrate and characterize its locomotion.

\section{A. Folding by Global Heating}

The key insight is to transform the internal stress of a contraction sheet into a folding torque. Our goal is to globally heat the structure to induce simultaneous foldings. The idea is inspired by the natural/artificial chemical compounds' folding, such as [23]. We wish to apply the general concept to different scales. Fig. 2 shows the designed mechanism for self-folding. We use a heat-sensitive contracting material

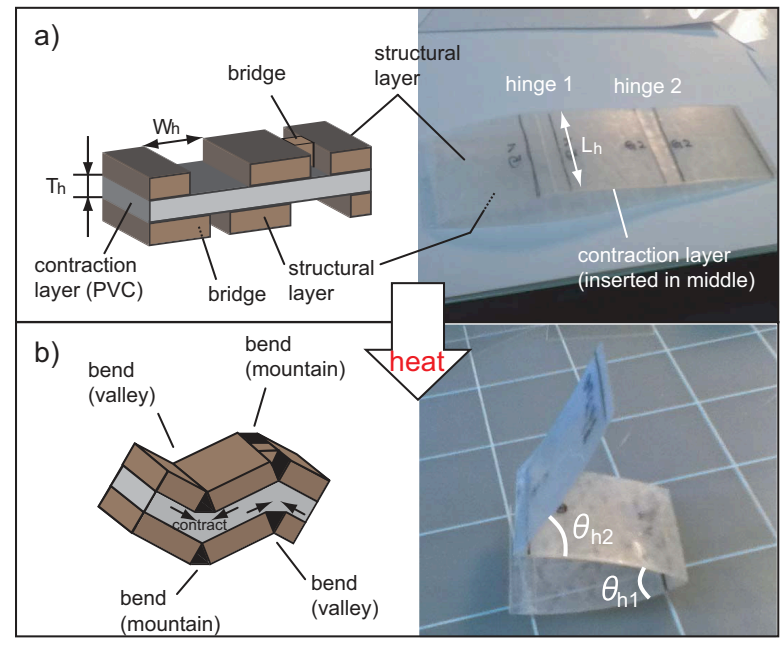

Fig. 2: Proposed self-folding method using global heating.

(polyvinyl chloride (PVC) Shrink Bag) for inducing shear force, which, in turn, creates the torque for bending a plane. The PVC contraction layer is sandwiched by nondeformable structural layers with different gap widths. Bending is controlled by designing gap widths in the structural layer. Here we demonstrate a particular design that achieves the transformation of a flat sheet into a completely enclosed cylinder. When the structure is heated, the shear force disproportionates to one side and generates a bending torque (Fig. 2(a),(b)). The gap width difference between the front and back of the sheet at the same position enables the differentiation of the bending direction. The approach is capable of folding mountain and valley folds simultaneously. Furthermore, the folding angle $\left(\theta_{h 1}\right.$ and $\theta_{h 2}$ in Fig. 2) can be approximately encoded with the gap widths. Namely, the wider $W_{h}$ becomes, the steeper the folding angle becomes.

PVC has two ideal features: the capability of low temperature contraction (less than $100{ }^{\circ} \mathrm{C}$ ), and a relatively robust physical structure with transparent visibility. PVC also has some disadvantages: the material naturally deforms, and it is relatively weak and subject to tearing.

The required torque $\tau_{h}$ for generating the elastic deformation of a hinge is generally given by [24]:

$$
\tau_{h}=E_{h} \frac{W_{h} \cdot T_{h}^{3}}{12 L_{h}} \theta_{h},
$$

where $E_{h}$ is the elastic modulus of the hinge, $W_{h}, T_{h}$, and $L_{h}$ are the length, width, and thickness of the hinge, respectively, and $\theta_{h}$ is the folded angle. For example, in Fig. 2 (b),(d), $\tau_{1}$ at hinge 1 is $1.48 \times 10^{-9} \mathrm{Nm}$ with values $E_{h}=3 \mathrm{MPa}$ (approximate assumption), $W_{h}=2.5 \mathrm{~mm}$, $T_{h}=0.03 \mathrm{~mm}, L_{h}=30 \mathrm{~mm}$, and $\theta_{h}=2.7 \mathrm{rad}$. The magnitude of the torque indicates that it is important to consider the weight of the structural layers when designing a large structure.

Our self-folding method is simple, it can be performed at low cost, and it is easily accessible using a home oven. Furthermore, the folding order could potentially be differentiated by incorporating more than one contraction material that responds to different temperatures.

\section{B. Precise Angle Control by V-fold Spans (Pop-up)}

The folding method for a precise folding angle of a flat sheet introduced in Section II-A is difficult due to the fact that we have two-dimensional material properties that have to be manipulated at the level of three-dimensional systems ${ }^{1}$. We focus on kinematically encodable folding angles that enable the control of a specific folding angle of a surface.

The folding is called a "V-fold" [26], whose one of the popular applications can be found in pop-up book art. Vfolds are known to achieve targeted folding angles by kinematically coupling with other folding angles (thus phrased "pop-up"). The transition from Fig. 3 (a) to 3 (b) describes an example of our approach. We aim at a mountain fold and a valley fold as described in Fig. $3(\mathrm{~b})$, respectively spanning $\theta_{\text {out } 1}$ and $\theta_{\text {out } 2},\left(\theta_{\text {out }} \in(0: \pi)\right)$. Here, mountain folds are shown in red lines, and valley folds are shown in blue lines. When all creases are folded simultaneously $\left(\theta_{\text {in }}: \pi \rightarrow 0\right)$, the attributed kinematics yield the sheet morphology shown in Fig. 3(b). The angles in Fig. 3 (a), $\alpha_{1}$ and $\alpha_{2}(\alpha \in(0: 2 \pi))$, designate the output angles $\theta_{\text {out } 1}$ and $\theta_{\text {out } 2}$ at respective $\theta_{i n}$. Moreover, the edge lengths $L_{H *}$ $(* \in[1,2,3])$ in Fig. 3 (b) can be encoded as the respective edge lengths in Fig. 3 (a), and the widths along the $x$-axis in 3 (b) as the combination with $L_{V}$ and the number of pleats

\footnotetext{
${ }^{1}$ One contrasted approach is so-called "thick origami approach [25]".
} 


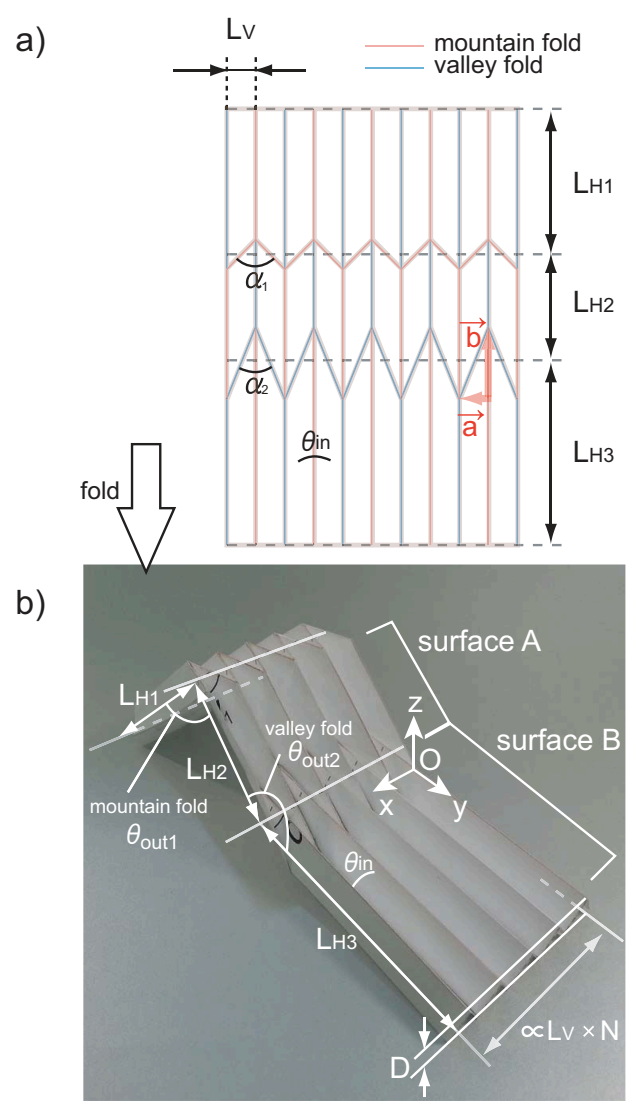

C)

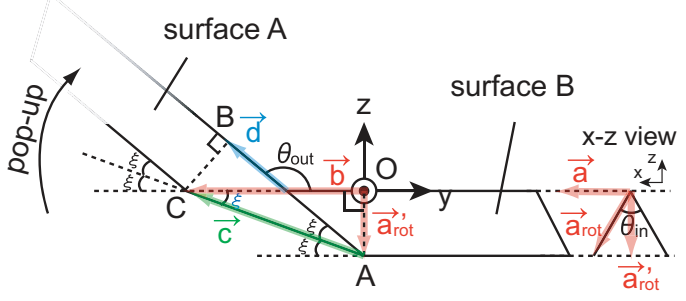

Fig. 3: Folding kinematics of V-fold. (a-b) Angle control by pleats accumulation. (c) Geometry of folding with respect to the input angle $\theta_{\text {in }}$ and the output angle $\theta_{\text {out }}$.

$N \in \mathbb{N}$. Namely $\propto N \times L_{V}$. Finally, the thickness of folded surfaces, $D$ in Fig. $3($ b), can be reduced by refining the resolution of pleats in accordance to $D \propto L$ ( $L$ is the scale length). In summary, the methodology is to roughly control $\theta_{i n}$ by the self-folding technique described in Section II-A, and obtain $\theta_{\text {out }}$ in precision ${ }^{2}$.

Fig. 3 (c) describes a side view of a valley V-fold, projected onto a y-z plane. We derive the relationship between $\theta_{\text {in }}$ and $\theta_{\text {out }}$ based on this schematic. We define constant vectors $\vec{a}$ and $\vec{b}$ shown in Fig. $3(\mathrm{a}),(\mathrm{c})$ as:

$$
\begin{aligned}
\vec{a} & =\left[\begin{array}{lll}
L_{v} & 0 & 0
\end{array}\right], \\
\vec{b} & =\left[\begin{array}{ll}
0 & -\frac{L_{v}}{\tan \frac{\alpha}{2}}
\end{array}\right] .
\end{aligned}
$$

\footnotetext{
${ }^{2}$ Although this work focuses on one-axial folds, various Origami works based on the V-fold imply significant room for attaining complex threedimensional shapes. However, these topics exceed the scope of this paper, and will be investigated in future work.
}

The rotation matrix along a unit vector $\vec{k}=[\kappa \lambda \mu]$ can be described using the rotation angle $\phi$ as:

$$
\begin{aligned}
& R(\kappa, \lambda, \mu, \phi)=\left[\begin{array}{c}
\cos \phi+\kappa^{2}(1-\cos \phi) \\
\lambda \kappa(1-\cos \phi)+\mu \sin \phi \\
\mu \kappa(1-\cos \phi)-\lambda \sin \phi
\end{array}\right. \\
& \kappa \lambda(1-\cos \phi)-\mu \sin \phi \quad \kappa \mu(1-\cos \phi)+\lambda \sin \phi] \\
& \cos \phi+\lambda^{2}(1-\cos \phi) \quad \lambda \mu(1-\cos \phi)-\kappa \sin \phi \\
& \left.\mu \lambda(1-\cos \phi)+\kappa \sin \phi \quad \cos \phi+\mu^{2}(1-\cos \phi)\right]
\end{aligned}
$$

With the input angle $\theta_{i n}\left(\theta_{i n}: \pi \rightarrow 0\right), \vec{a}_{r o t}$, which is the tangent vector along the plane is:

$$
\vec{a}_{\text {rot }}=\vec{a} R\left(0,-1,0, \frac{\pi-\theta_{i n}}{2}\right) .
$$

The projection of $\vec{a}_{r o t}$ onto the y-z plane $\vec{a}_{r o t}^{\prime}$ is:

$$
\vec{a}_{\text {rot }}^{\prime}=\vec{a}_{\text {rot }}\left[\begin{array}{ccc}
0 & 0 & 0 \\
0 & 1 & 0 \\
0 & 0 & 1
\end{array}\right] .
$$

Since $\vec{c}=\vec{b}-\vec{a}_{\text {rot }}^{\prime}$, and considering that $\triangle \mathrm{OAC}$ and $\triangle \mathrm{BCA}$ are homologous, $\vec{d}$ can be obtained by rotating $\vec{c}$ for angle $\xi$, and adjusted to a certain length by scaling by a constant $s(s \in \mathbb{R})$ as:

$$
\vec{d}=s \vec{c} R(-1,0,0, \xi),
$$

where $\xi=\arccos \frac{\vec{b} \cdot \vec{c}}{|\vec{b}||\vec{c}|}$. The targeted output angle $\theta_{\text {out }}$ can be derived by measuring the angle between $\vec{b}$ and $\vec{d}$ as:

$$
\theta_{\text {out }}=\arccos \frac{-\vec{b} \cdot \vec{d}}{|-\vec{b}||\vec{d}|} .
$$

We define the completion of a V-fold as:

$$
\text { completion }[\%]=\frac{\pi-\theta_{\text {out }}}{\pi-\left.\theta_{\text {out }}\right|_{\theta_{\text {in }}=0}} \times 100,
$$

as an indication of the accomplishment degree of a targeted folding (pop-up) angle.

Fig. 5 (a) shows the relation between $\theta_{i n}$ and completion with different $\alpha$ varying from $\alpha=30^{\circ}$ to $\alpha=150^{\circ}$ in 5 levels. It can be seen that the convergence of completion has good speeds: $80 \%$ of the folding angles are already accomplished at $\theta_{i n} \approx 90^{\circ}$, especially when $\alpha>90^{\circ}$. Since actual convergence of $\theta_{\text {out }}$ when self-folding is determined by the physical factors (e.g., applied heat temperature, internal stress of PVC, and the gap widths of hinges), it settles at $\theta_{\text {in }}<90^{\circ}$ (finely-tunable). Fig. 5 (a) shows that the larger $\alpha$ becomes, the faster it folds. By considering these physical tendencies, the actual folding angles can be pre-adjusted by setting wider $\alpha$ to obtain precise folding angles $\theta_{\text {out }}$.

Fig. 5 (b) shows the angle size $\theta_{\text {in }}$ required to gain a desired completion for different $\alpha$. Attaining smaller $\theta_{\text {in }}$ requires more compression of the contraction layer and, thus, requires more heat energy input. The data shows that the larger $\alpha$ is, the less energy is required. Also, $70 \%$ completion can be attained for most $\alpha$ even if $\theta_{\text {in }}$ does not reach less than $90^{\circ}$. 


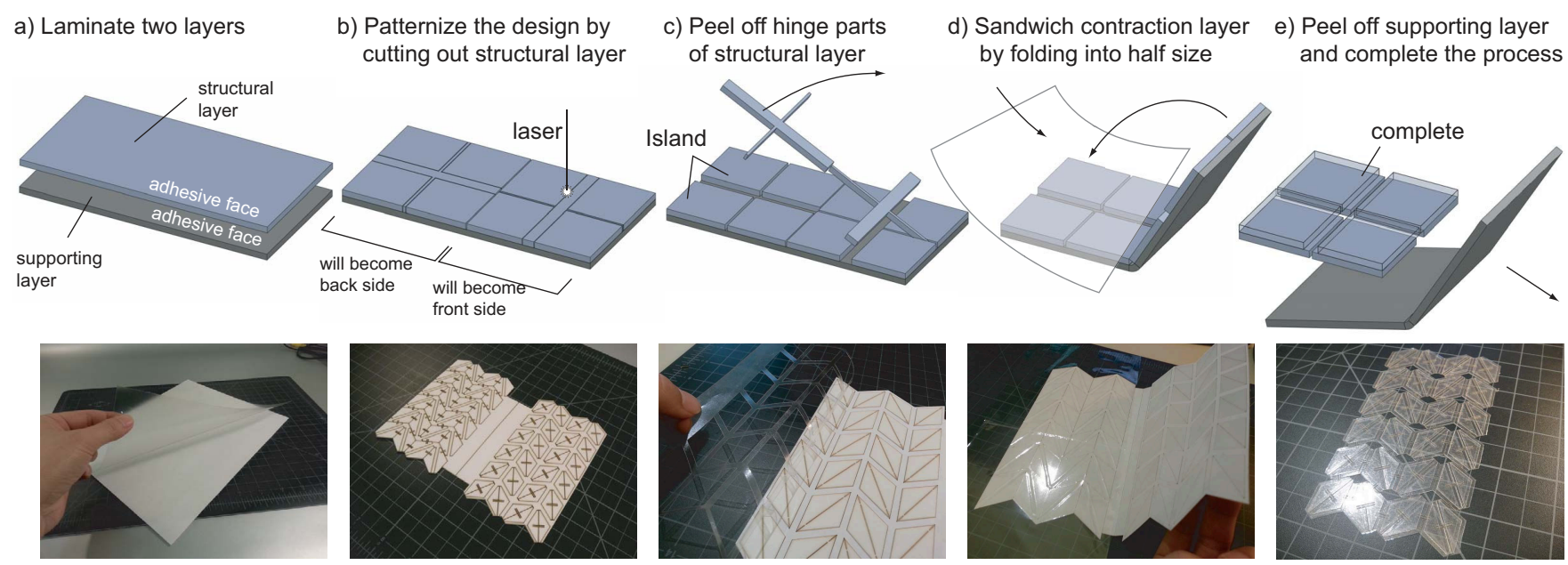

Fig. 4: Fabrication process of self-folding multi-creased pattern. Structural materials are placed in designed gap widths in the front and back of the contraction layer.

(a)

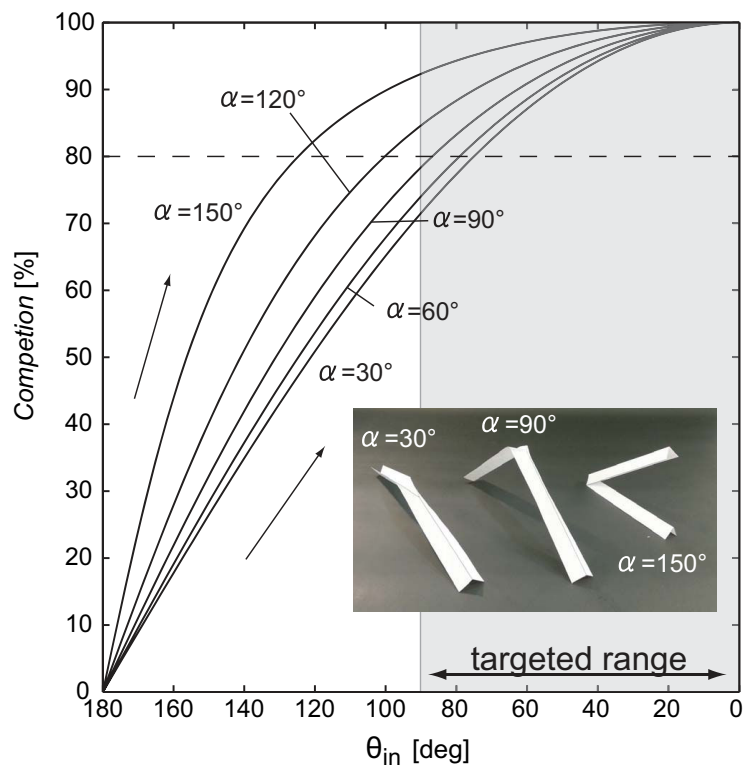

(b)

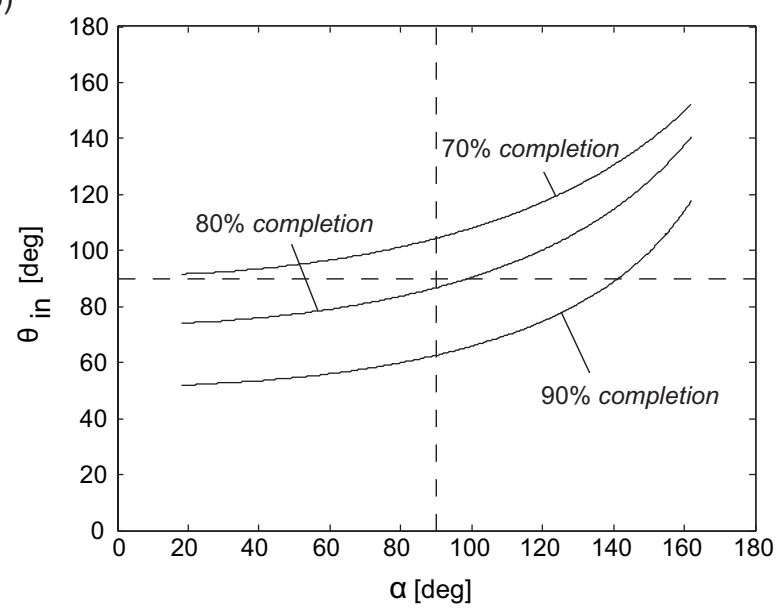

Fig. 5: (a) Convergence of $\theta_{\text {out }}$ in correspondence to $\theta_{\text {in }}$ with different $\alpha$. (b) $\theta_{\text {in }}$ required to gain a desired completion for different $\alpha$.

\section{Fabrication of Self-folding Crease Pattern}

Manually folding a multi-crease structure is a time consuming process. When the folding steps are on the order of dozens, or multiple creases need to be folded simultaneously, self-folding can greatly reduce the entire fabrication time. In this section, we introduce a fabrication method for selffolding that is simple and fast, but also accurate and easily reproducible. The key insight is that the folding directions are determined by the gap width differences of the hinge parts (Section II-A). The methodology is to sustain both the front and back side of structural materials' relative positions on a single supporting sheet, and sandwich a contraction layer by folding in half, such that corresponding gaps come on top and below the contraction layer (Fig. 4).

First, the supporting layer (Post-it Sheet Labels and Permanent Adhesive Full Sheet Labels, 3M) and the structural layer (Ink Jet Adhesive Film, Grafix, thickness: $0.12 \mathrm{~mm}$ ) are laminated, such that the adhesive face of the structural layer faces out (Fig. 4(a)). Using a laser cutting machine (VersaLASER, VLS 3.50) with regulated laser strength, we cut only through the structural layer and trim the front and back patterns (Fig. 4(b)). The frame-like segments of structural layers that cover the hinge parts are peeled off (Fig. 4(c)). A contraction layer (PVC, thickness: $0.03 \mathrm{~mm}$ ) is inserted and sandwiched by folding the structural layer in half, such that the respective hinges overlap above and below the contraction layer (Fig. 4(d)). We manually cut out the intersections of hinges using a cutter, where 6 creases are gathered. This process was incorporated due to some bending direction errors we often encountered when heating up. However, further investigation is still required to conclude if this step is necessary. We peel off the supporting layer, and obtain the complete structure (Fig. 4(e)). The complete structure has a thickness of $0.27 \mathrm{~mm}$ with the size of $70 \times 125 \mathrm{~mm}^{2}$. This fabrication method has an advantage with forms where large scale shapes are represented by iterative crease accumulations. 

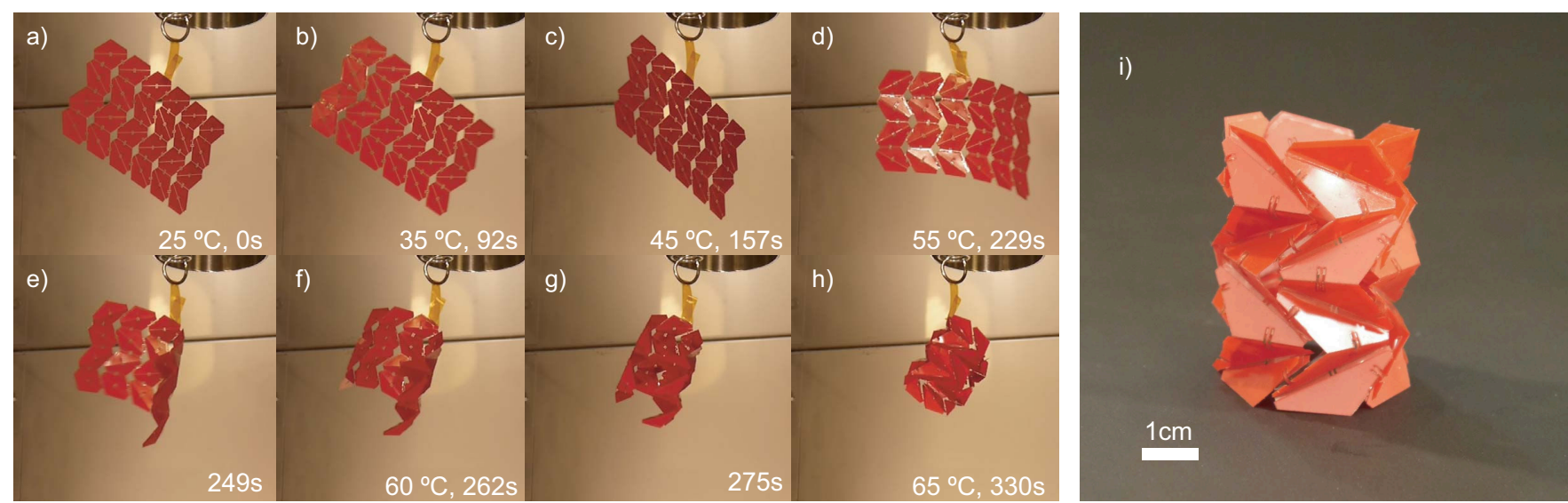

Fig. 6: Self-folding process attained by baking in a heat oven.

\section{SElf-Folding And PROOF OF CONCEPT IN ROBOTICS}

In this section, we show how our self-folding method can be used to make a mobile robot. We show that the function of a self-folded skeleton as a stabilizer of locomotion, and perform a basic locomotion capability, coupled with an easily implementable encapsulated actuation unit (Fig. 1).

\section{A. Structure Design for Self-folded Cylindrical Robot}

The outer skeleton of the robot is a hollow cylindrical structure with symmetry, simplicity, and postural robustness. The pleat pattern, the $\mathrm{X}$-form span, is one of the most complex foldings among V-folds, where intersections gather six creases, thus posing a significant self-folding challenge.

Fig. 7 (a) shows two V-folds (colored in red) where the spanning angles are $\alpha$ interleaving a valley fold (colored in blue). For the formation of the $3 \mathrm{D}$ structure, we aim at
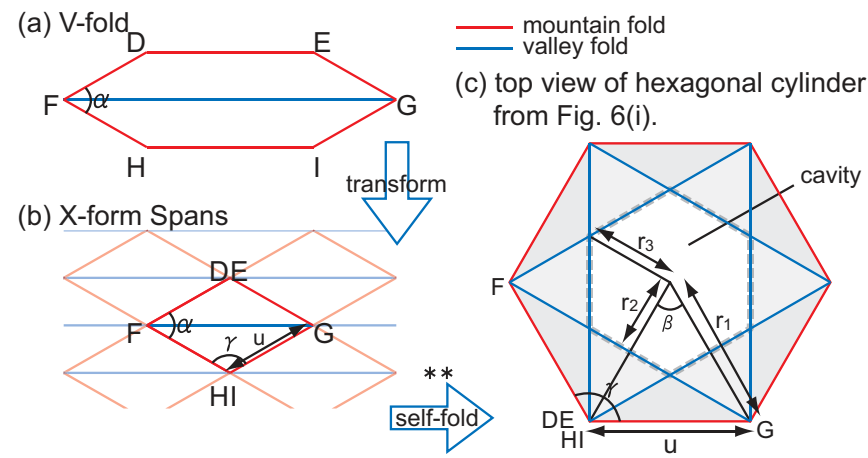

Fig. 7: X-form Spans which is an extension of the V-fold; (a) Vfold, (b) The X-form Spans as an extension of the V-fold, and (c) side view after complete folds. ** Alternate the direction of every second row.

a cylindrical shape using the $\mathrm{X}$-form Spans, which can be regarded as an extension of the V-fold (Fig. 7 (b)). The Xform Spans can be derived by transforming the V-fold; from 7 (a), we shorten the distances D-E and H-I, and configure a rhombus. When arrays of this rhombus are folded, a regular $n$-sided polygonal cylinder is generated (the hexagonal case is shown in Fig. 7 (c) in top-view). Two designing parameters, $\alpha$ and $u$, can be determined by examining the geometry of the hollow cavity that will be created in the folded $n$ sided polygonal structure. When the geometric parameters of the actuation unit $r_{2}, r_{3}$, and $n$ are given, these designing parameters are determined as:

$$
\begin{aligned}
\alpha & =\frac{\pi}{2}-\frac{n-2}{n} \pi, \\
u & =\frac{2 \sin \frac{\pi}{n}}{\cos \frac{2 \pi}{n}} \times r_{2}, \\
& =\tan \frac{2 \pi}{n} \times r_{3} .
\end{aligned}
$$

Instead of a rhombus X-form Spans, more general models of origami-inspired polygonal cylinders have been investigated in [27], [28]. The size of the body and the resolution of pleats can vary. We selected a hexagonal shape because the resulting folded outer skeleton has sufficient volume to include all the robot's components, at the same time the flat patches provide support and friction for locomotion. our designed robot will use the flat areas to contact the ground and control slippage. The arrays of rhombuses are alternately redirected, such that when the sheet folds, zigzagshaped edges naturally latch and, thus, the structure is stable (see Fig. $2(\mathrm{~g})$ ). We set $\alpha=60^{\circ}, u=20 \mathrm{~mm}$, gap width $=2.5 \mathrm{~mm}$, bridge width $=1 \mathrm{~mm}$. The designed shape consists of 62 creases that must be folded simultaneously, thus, difficult to fold by hand.

\section{B. Self-folding Process}

Fig. 6 shows the self-folding process attained in a heat oven. In order to transmit uniform heat to the sheet, the sheet was hung from the ceiling. This setting contributes to preventing direct physical contact from the heat source (generally the bottom of oven). The oven we employed blows air from the side wall and, thus, the sheet swings during the process $^{3}$.

\footnotetext{
${ }^{3}$ The air blow is an intrinsic functionality of the oven that we could not stop; however, we assume the influence of the blow is not essential judging from other smaller scale trials in another normal oven.
} 
We ramped the temperature from room temperature $\left(25^{\circ} \mathrm{C}\right.$ ) to $65^{\circ} \mathrm{C}$ (Fig. 6(a)). When the temperature reached approximately $55^{\circ} \mathrm{C}$, the crease pattern appeared on the sheet's surface, increasing the sculpture, and forming a global curve (Fig. 6(d)-(g)). When the temperature reached $65{ }^{\circ} \mathrm{C}$, the structure formed a complete cylindrical shape, automatically latching both sides via zigzag tooth-shaped edges (Fig. 6(h)). The process was completed in approximately 5 minutes. The duration depends on the capability of the oven, as well as the size of the materials, and should be optimized according to conditions.

Note that the configured shape is made of 62 folds; that is, the failure rate of an individual fold is maximally less than $1 / 62 \times 100=1.61 \%$. We have attempted 4 trials and encountered no error. As for a unique discovery found in previous failure models, it is required that all 62 folds be completed without a failure. Should even one fold fail, the global structure shapes as a distorted flat sheet.

\section{Mechatronics}

A modularized actuation unit, which shapes the hexagonal cylinder to fit to the outer skeleton, is developed. The actuation unit features collaterally assigned vibration motors for locomotion. By alternating the movement of vibration motors, the robot can move to any planar configuration, inspired by [29]-[31] (Fig. 8(b)). The controller board was developed on a printed flexible circuit, whose folded geometry fits into the actuation unit. Finally, the actuation unit is manually inserted and attached to the outer skeleton. The projecting nails hold the position of the actuation unit to reside stably in the outer skeleton.

The circuit is printed onto a sheet layer and then folded. The circuit substrate is composed of three sheet materials: one with rigid stiffness used as a backbone layer (polystyrene), another with heat resistive properties (polyimide), and the third with conductivity (copper). After these

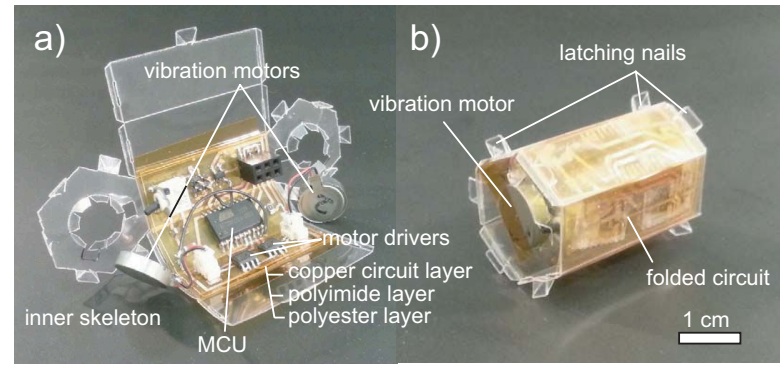

Fig. 8: Encapsulated actuation unit. (a) Self-folded inner-skeleton and the flexible circuit with components. (b) Folded flexible circuit into hexagonal cylindrical shape.

layers are laminated into one sheet, coating material is sputtered onto the copper layer in the shape of a circuit using a solid-ink printer. The remaining intact copper material is then etched being soaked in Ferric Chloride for about 15 minutes, and is scrubbed away. Slits are added using a cutter, and bent manually to fit into the inner skeleton that was previously cut by laser cut machine and folded. The
TABLE I: Components used in the robot

\begin{tabular}{ccc}
\hline Device & Weight $[\mathrm{g}]$ & Type \\
\hline \hline Outer skeleton & 1.966 & self-folded \\
MCU & 0.561 & ATiny2313A, Atmel \\
Vibration motor & $1.390 \times 2$ & $1.8 \mathrm{G}, \mathrm{T} . P . C$. \\
Flexible circuit layer & 0.442 & printed on 3 layers \\
Other SMD components & 0.283 & switch, etc. \\
Connectors & 1.526 & ISP Connectors, etc. \\
LiPo battery & 1.773 & 40 mAh, Great Power Battery \\
Inner skeleton and others & 1.428 & cage, cables, solder \\
\hline total weight & 10.759 &
\end{tabular}

fabricated layer is resistant to heat, but is also lightweight at $0.442 \mathrm{~g}$. We list the components used in the robot in Table I.

The controller consists of a micro control unit (MCU), two vibration motors with respective motor drivers, electricity regulating components, a battery, and other accessories, such as connectors. We selected the ATtiny 2313A for the low operation voltage, and for serial communication capability for future use. Since the circuit becomes the part of the robot, weight distribution of components was considered and designed.

Fig. 9 shows the weight distribution plotted in a pie chart. The body (outer skeleton) only weighs $18.27 \%$, which is

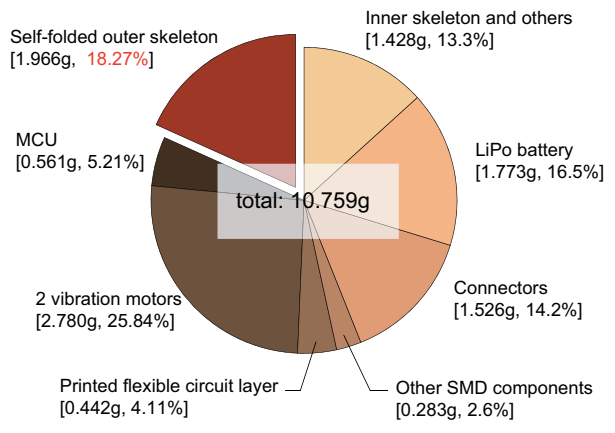

Fig. 9: Weight distribution of the robot.

remarkably lightweight, and shows the merits of forming a body through foldings.

\section{Results}

Fig. 10 shows the demonstrated locomotions. Control of the actuators are managed by an open-loop control, whose driving current is managed by Pulse Width Modulation (duty ratio $=50 \%$ ). It can be recognized that both translational (Fig. $10(\mathrm{a})$ ) and rotational (Fig. 10 (b)) motions are demonstrated by differentiating the direction of rotations of vibration motors. When moving forward, we turn both motors in the same direction, and the translational locomotion speed is $2.95 \mathrm{~cm} / \mathrm{s}$. When turning, the motors turn in opposite directions, and the robot's turning angular speed is $0.74 \mathrm{rad} / \mathrm{s}$ $\left(42.4^{\circ} / \mathrm{s}\right)$. However, especially for the translational motion, the motion is sensitive to the friction of the ground, and is not necessarily showing constant speed when changing the motor speeds. We also assume that the asymmetric pleat patterns will have an influence on the stability of motion. 


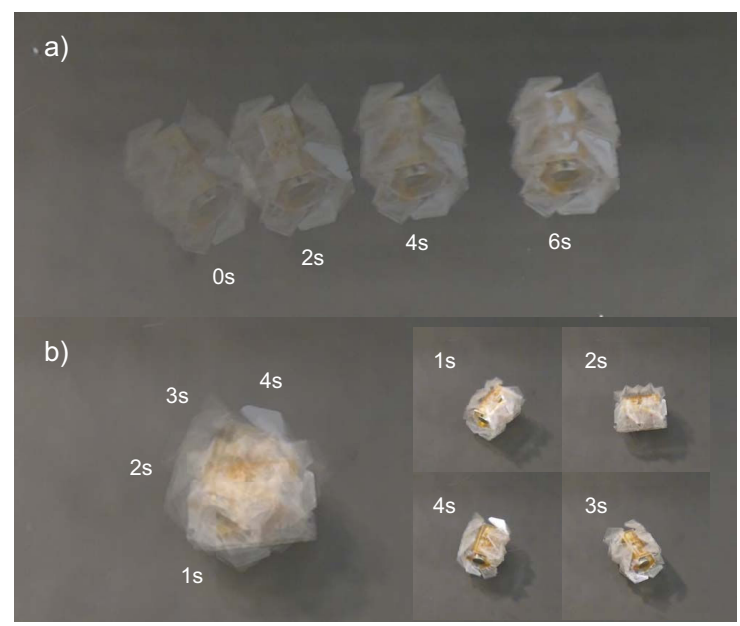

Fig. 10: Results of motions. (a) Translational motion by turning two motors in the same direction. (b) Rotational motion by turning in opposite directions.

\section{CONCLUSION}

This paper presents a scheme for self-folding for the autonomous fabrication of an origami robot using global heat. We first develop a method that enables autonomous and simultaneous folds of mountain and valley creases in a sheet structure. We then focus on the characteristics of the V-fold Spans where one of the angles can be precisely controlled by kinematically coupling it to another angle. The X-form Spans mechanism is then incorporated as an extension of V-fold. Using uniform heat on the designed sheet in this fashion, a cylindrical robot body is attained. We developed an easy, fast, and reliable fabrication technique for constructing the self-folding sheet. The self-folding process completes in approximately 5 minutes, where 62 folds are simultaneously managed at $65^{\circ} \mathrm{C}$. We further develop a mobile robot using this self-folded body. The robot has two vibration motors for locomotion, caged by the self-folded outer skeleton for stabilization. The robot locomotion proves the functionality of self-folded origami structure.

\section{REFERENCES}

[1] T. Fukuda and Y. Kawauch, "Cellular robotic system (CEBOT) as one of the realizations of self-organizing intelligent universal manipulator," in IEEE International Conference on Robotics and Automation (ICRA), 1990, pp. 662-667.

[2] M. Yim, "New locomotion gaits," in IEEE International Conference on Robotics and Automation (ICRA), 1994, pp. 2508-2514.

[3] K. Nakano, S. Uchihashi, N. Umemoto, and H. Nakagama, "An approach to evolutional system," in First IEEE Conference on Evolutionary Computation (CEC), 1994, pp. 781-786.

[4] K. Kotay, D. Rus, M. Vona, and C. McGray, "The self-reconfiguring robotic molecule," in IEEE/RSJ International Conference on Intelligent Robots and Systems (IROS), 1998, pp. 424-431.

[5] S. Murata, K. Tomita, E. Yoshida, H. Kurokawa, and S. Kokaji, "Self-reconfigurable robot," in International Conference on Intelligent Autonomous Systems (IAS), 1999, pp. 911-917.

[6] S. Griffith, D. Goldwater, and J. Jacobson, "Self-replication from random parts," Nature, vol. 437, p. 636, 2005.

[7] E. Klavins, "Programmable self-assembly," IEEE Control System Magazine, vol. 27, pp. 43-56, 2007.

[8] S. Miyashita, M. Göldi, and R. Pfeifer, "How reverse reactions influence the yield rate of stochastic self-assembly," International Journal of Robotics Research, vol. 30, pp. 627-641, April 2011.
[9] G. Mermoud, M. Mastrangeli, U. Upadhyay, and A. Martinoli, "Realtime automated modeling and control of self-assembling systems," in IEEE International Conference on Robotics and Automation (ICRA), 2012, pp. 4266-4273.

[10] K. C. Cheung, E. D. Demaine, J. R. Bachrach, and S. Griffith, "Programmable assembly with universally foldable strings (moteins)," IEEE Transactions on Robotics, vol. 27, pp. 718-729, 2011.

[11] L. Brodbeck and F. Iida, "Enhanced robotic body extension with modular units," in IEEE/RSJ International Conference on Intelligent Robots and Systems (IROS), 2012, pp. 1428-1433.

[12] A. M. Hoover, E. Steltz, and R. S. Fearing, "RoACH: An autonomous $2.4 \mathrm{~g}$ crawling hexapod robot," in IEEE/RSJ International Conference on ntelligent Robots and Systems (IROS), 2008, pp. 26-33.

[13] K. L. Hoffman and R. J. Wood, "Towards a multi-segment ambulatory microrobot," in IEEE International Conference on Robotics and Automation (ICRA), 2010, pp. 1196-1202.

[14] J. Whitney, P. Sreetharan, K. Ma, and R. Wood, "Pop-up book mems," Journal of Micromechanics and Microengineering, vol. 21, no. 11, p. 115021, 2011.

[15] C. D. Onal, R. J. Wood, and D. Rus, "Towards printable robotics: Origami-inspired planar fabrication of three-dimensional mechanisms," in IEEE International Conference on Robotics and Automation (ICRA), 2011, pp. 4608-4613.

[16] L. Ionov, "Soft microorigami: self-folding polymer films," Soft Matter, vol. 7, pp. 6786-6791, 2011.

[17] E. Hawkes, B. An, N. M. Benbernou, H. Tanaka, S. Kim, E. D Demaine, D. Rus, and R. J. Wood, "Programmable matter by folding," Proceedings of the National Academy of Sciences, vol. 107, no. 28, pp. 12 441-12 445, 2010.

[18] S. M. Felton, M. T. Tolley, C. D. Onal, D. Rus, and R. J. Wood, "Robot self-assembly by folding: A printed inchworm robot," in IEEE International Conference on Robotics and Automation (ICRA), 2013, pp. 277-282.

[19] J. Ryu, M. DAmato, X. Cui, K. N. Long, H. J. Qi, and M. L. Dunn, "Photo-origamibending and folding polymers with light," Applied Physics Letters, vol. 100, pp. 161908-1-5, 2012.

[20] Y. Liu, J. K. Boyles, J. Genzer, and M. D. Dickey, "Self-folding of polymer sheets using local light absorption," Soft Matter, vol. 8, pp. 1764-1769, 2011

[21] R. V. Martinez, C. R. Fish, X. Chen, and G. M. Whitesides, "Elastomeric origami: Programmable paper-elastomer composites as pneumatic actuators," Advanced Functional Materials, vol. 22, p. 13761384, 2012.

[22] J. Guan, H. He, D. J. Hansford, and L. J. Lee, "Self-folding of three-dimensional hydrogel microstructures," The Journal of Physical Chemistry B, vol. 109, pp. 23 134-23 137, 2005.

[23] P. W. K. Rothemund, "Folding DNA to create nanoscale shapes and patterns," Nature, vol. 440, no. 7082, pp. 297-302, 2006.

[24] E. Iwase and I. Shimoyama, "Multistep sequential batch assembly of three-dimensional frerromagnetic microstructures with elastic hinges," Journal of Microelectromechanical Systems, vol. 14, pp. 1265-1271, 2005.

[25] T. Tachi, "Rigid-foldable thick origami," in Origami 5: the 5th International Conference on Origami in Science Mathematics and Education, 2010

[26] P. Jackson, Folding Techniqus for Designers From Sheet to Form, P. Jones, Ed. Laurence King Publishing Ltd, 2011.

[27] G. W. Hunt and I. Ario, "Twist buckling and the foldable cylinder: an exercise in origami," International Journal of Non-Linear Mechanics, vol. 40, pp. 833-843, 2005.

[28] C. C. Min and H. Suzuki, "Geometrical properties of paper spring," in Manufacturing Systems and Technologies for the New Frontier, 2008, pp. $159-162$.

[29] K. Ioi, "A mobile micro-robot using centrifugal forces," in IEEE/ASME International Conference on Advanced Inelligence Mechatronics, 1999, pp. 736-741.

[30] S. Miyashita, F. Casanova, M. Lungarella, and F. Rolf Pfeifer, "Peltierbased freeze-thaw connector for waterborne self-assembly systems," in IEEE/RSJ International Conference on Intelligent Robots and Systems (IROS), 2008, pp. 1325-1330.

[31] M. Rubenstein, C. Ahler, and R. Nagpal, "Kilobot: A low cost scalable robot system for collective behaviors," in IEEE International Conf on Robotics and Automation (ICRA), 2012, pp. 3293-3298. 